\title{
Concurrence chez Vicia sativa L et Avena sativa L. II. Effets des contraintes hydriques et nutritionnelles sur la biomasse et la distribution des ressources
}

\author{
M Ouknider $1,{ }^{*}$, P Jacquard 1, D Elkhyari 2 \\ 1 CNRS, Centre Louis Emberger, BP 5051, 34033 Montpellier, France \\ 2 Université Mohamed $V$, faculté des sciences, département de biologie, Rabat, Raroc
}

(Reçu le 24 août 1990, accepté le 23 septembre 1991)

\begin{abstract}
Résumé - La variation des effets d'interférence entraînés par la présence de l'avoine sur la croissance de la vesce et réciproquement est étudiée. Ces effets sont exprimés en biomasse et en répartition de cette dernière entre les différents organes. Quand les rendements en poids sec produits par la vesce et l'avoine en association sont comparés avec les productions correspondantes en culture pure, à densité numérique équivalente, la production par plante d'avoine associée est augmentée et celle de la vesce associée est diminuée par la concurrence interspécifique. La diminution du potassium dans la solution nutritive a un effet d'autant plus marqué que le niveau d'azote est élevé. En conditions de déficience en potassium, la distribution des ressources aux racines augmente au détriment des feuilles. En association, la répartition de la biomasse varie entre les 2 espèces : augmentation de la proportion affectée aux tiges et aux racines chez la vesce, diminution de la part racinaire et augmentation de la proportion foliaire chez l'avoine.
\end{abstract}

association vesce-avoine / biomasse / $\mathrm{K}$ / concurrence

Summary - Competition in Vicia sativa $L$ and Avena sativa $L$. II. Effects of water and nutritional stresses on biomass and allocation of resources. The variation in interference effects of oats on the growth of vetch and viceversa is studied. This variation is expressed in terms of biomass and resource allocation to the organs. Vetch dry weight per plant increases more when seeded alone than in mixture. Oats, however, shows an opposite trend (fig 1). The growth curves of vetch and oats in association have been compared with the curves in pure stand at half-density (fig 2). The 2 species have the same response to a reduction in water resource (fig 3). The decrease of potassium in the nutrient solution has an effect on biomass/plant, increasing with the level of nitrogen (fig 4). In case of $K$ deficien$c y$, resources allocated to roots increase, mainly at the expense of leaves (fig 5). In the mixture, dry weight partitioning pattern is different for the 2 species: there is an increase in the proportion allocated to stems and roots for vetch and a decrease in the proportion allocated to roots and an increase in the proportion allocated to leaves for oats (fig 6).

vetch-oat mixture / biomass / $\mathrm{K} / \mathrm{N}$ / competition

\section{INTRODUCTION}

Plusieurs travaux ont montré que les interactions compétitives et les effets de voisinages sont des facteurs déterminants dans les stratégies adaptatives des plantes (Grime, 1977), pour la cohabitation des espèces (Turkington et
Harper, 1979; Fowler, 1982), leur distribution (Werner et Platt, 1976) et leur diversité (Pickett et Bazzaz, 1978; Peterson et Bazzaz, 1978).

Par sa nature, la compétition est un processus actif dont le résultat dépend de l'aptitude relative des espèces à prélever et à utiliser les ressources disponibles; elle se traduit par une modification de l'environnement immédiat.

\footnotetext{
* Adresse actuelle : ENA, département d'agronomie, BPS 40, Meknès, Maroc
} 
Les différentes espèces répondent à ces variations en modifiant l'efficience de leurs mécanismes d'acquisition des ressources et l'utilisation interne de leurs ressources. La croissance dépend de la capacité à réaliser ces modifications et conditionne le succès écologique et évolutif, ou le rendement agronomique. La répartition des ressources entre les différents organes (répartition entre parties aériennes et racines, par exemple) représente un compromis d'utilisation des ressources, puisque chaque ressource limite les différentes fonctions du végétal. De nombreuses expériences où l'équilibre entre ressources est modifié tendent effectivement à montrer qu'il y a un changement du rapport racines/parties aériennes en faveur de l'organe qui permet d'acquérir la ressource la plus limitante. L'effet d'une déficience en azote sur la répartition de biomasse entre parties aériennes et souterraines semble être le même que celui provoqué par une diminution conjointe de tous les éléments minéraux (Troughton, 1977). Sur trèfle violet, Jacquard et al (1985) ont montré que l'investissement des ressources s'effectue toujours au profit des pétioles et des limbes du premier ordre, et au détriment des tiges et des racines. Une insuffisance nutritionnelle du milieu modifie, à âge foliaire égal, la répartition des assimilats : la proportion consacrée aux tiges devient nulle en situation de carence.

Sur trèfle violet associé au ray-grass, Thomas (1984) a montré que le déficit hydrique réduit la croissance, diminue la proportion de feuilles et augmente celle des stolons. Watkinson (1982), sur Vulpia fasciculata, a rapporté que l'effet du régime hydrique, pour une densité donnée, est plus marqué en culture pure qu'en association.

L'importance de la répartition des assimilats sur la croissance a été évaluée par Winner et al (1985) en comparant des radis sauvages et cultivés : les vitesses de photosynthèse des 2 espèces sont égales, mais après 5 semaines de croissance avec des minéraux non limitants, la biomasse de l'espèce sauvage est double de celle de l'espèce cultivée. Chez cette dernière, le rapport de répartition entre parties souterraines et parties aériennes est de 1,99 contre 0,31 chez l'espèce sauvage.

Dans un précédent article (Ouknider et al, 1991) nous avons décrit les paramètres morphogénétiques (rythme d'apparition et durée de vie des feuilles, tallage, ramification) de la vesce et de l'avoine en conditions de compétition intra et interspécifique. Les résultats obtenus font ressortir que lorsque les plantes interfèrent en asso- ciation comme en culture pure (effet densité), la concurrence agit de façon différente sur le rythme d'émergence foliaire selon l'espèce (vesce ou avoine). Des différences de phyllochrone entre les 2 espèces peuvent entraîner un établissement très décalé dans le temps, des surfaces foliaires qui interceptent l'énergie lumineuse. En conséquence, l'occupation de l'espace aérien spécifique à chaque composant se traduit alors par une compétition pour la lumière. Ainsi, la carence en potassium augmente le phyllochrone des 2 espèces et le taux d'émergence foliaire présente une interaction importante avec les niveaux d'azote et de potassium. Le présent article étudie les variations de la biomasse, qui intègrent les modifications morphogénétiques, et de sa répartition entre les différents organes (feuilles, tiges et racines), sous l'effet de la concurrence.

\section{MATÉRIEL ET MÉTHODES}

Les résultats qui sont présentés se rapportent aux 3 dispositifs dont le matériel végétal et les techniques ont été détaillés dans la première partie de cette étude (Ouknider et al, 1991) :

- expérience 1, portant sur l'effet de 4 densités $(8,16$, 24 et 40 individus par pot) dans un dispositif placé à l'extérieur et irrigué par un système goutte à goutte;

- expérience 2, conduite en hydroponie, sur sable siliceux, en serre, à la densité de 8 plantes par pot et selon un dispositif factoriel comportant 3 niveaux de $\mathrm{K}$ $(0,6 \mathrm{mN}, 0,3 \mathrm{mN}, 0,1 \mathrm{mN})$ et 2 niveaux d'azote $(1,4 \mathrm{mN}, 0,3 \mathrm{mN})$;

- expérience 3 , installée sous serre, avec 4 densités : $4,8,16$, et 32 individus par pot, soumises à 3 régimes hydriques $\left(H_{1}\right.$, non limitant proche de la capacité au champ, $\mathrm{H}_{2}$, intermédiaire, $\mathrm{H}_{3}$, fortement contraignant).

Toutes ces expériences comportaient les cultures pures des 2 espèces et leur association dans la proportion 1/1. C'est uniquement pour l'expérience 2 que la distribution de la biomasse entre les différents organes est envisagée. Par contre, seule la biomasse aérienne est présentée pour les expériences 1 et 3.

\section{Prélèvement des échantillons}

Les plantes de l'ensemble des unités expérimentales correspondant aux traitements testés (expérience 2) ont été récoltées après $40 \mathrm{j}$ de croissance. Les racines ont été soigneusement séparées du sable par immersion dans de l'eau déminéralisée de façon à ne pas briser les radicelles, et rincées rapidement à plusieurs reprises, essorées, puis épongées avec du papier filtre. Sur chaque plante, on a séparé les organes sui- 
vants : racines, feuilles, tiges et inflorescences. Ces parties ont été mises à sécher à $80^{\circ} \mathrm{C}$. Elles furent ensuite pesées sur une balance de précision. Pour chaque organe quand celui-ci était présent, on a estimé la distribution de la matière sèche, en calculant le rapport du poids sec de l'organe sur le poids sec total de l'individu. On exprime ainsi la répartition entre différents organes, en terme de matière sèche. Les travaux de Hickman et Pitelka (1975) montrent qu'il n'existe pas de différence entre les modèles de répartition basés sur des estimations calorimétriques (investissement énergétique) et ceux établis sur la biomasse sèche.

\section{Modèle mathématique de réponse à la compétition}

La biomasse par individu $\left(w_{i}\right)$ est une fonction linéaire des densités respectives $\left(d_{j}\right.$ et $d_{j}$ ) des 2 composants (Connolly, 1987) :

$$
\begin{aligned}
& w_{i}=a_{i 0}+a_{i i} d_{i}+a_{i j} d_{j} \\
& w_{j}=a_{j o}+a_{i j} d_{j}+a_{j i} d_{i}
\end{aligned}
$$

où $a_{i i}$ et $a_{i j}$ sont les coefficients intraspécifiques mesurant l'effet de la densité d'une espèce sur elle-même; $a_{i j}$ et $a_{i j}$ sont les coefficients interspécifiques mesurant l'effet de la densité d'une espèce sur l'autre. Les effets relatifs des 2 espèces sont mesurés par leurs taux de substitution $a_{i j} / a_{i j}$ et $a_{i j} / a_{i j}$, lesquels sont inférieurs à 1 si la compétition intraspécifique est prépondérante, supérieurs à 1 si la compétition interspécifique est prépondérante et égaux à 1 si les deux compétitions sont de même intensité.

\section{RÉSULTATS}

\section{Biomasse des plantes de vesce et d'avoine}

\section{Effet de l'association et de la densité}

L'aptitude à la compétition peut être estimée à densité égale de semis par la comparaison entre les croissances en association et en culture pure. Les courbes représentées dans la figure 1 illustrent la variation de la biomasse par plante des 2 espèces, avoine pure et associée d'une part ( $a$ et b) et vesce pure et associée d'autre part (c et d) sous l'effet de la densité. On s'aperçoit que quel que soit le stade considéré, la biomasse par plante se classe en sens inverse de la densité. En culture associée, la matière sèche par plante présente le même comportement. La contrainte ainsi créée s'accentue avec l'âge, à partir du moment où les plantes entrent en compétition avec leurs voisines. Il en résulte une réduction dans la vitesse de croissance d'autant plus marquée que la densité augmente.

Les effets d'interférence produits sur la croissance des vesces par la présence des avoines pendant toute la durée de la culture montrent que, quand le rendement en poids sec produit par la vesce et l'avoine en association sont comparés à leurs productions correspondantes en culture pure, à densité de semis équivalente, la production par plante d'avoine est augmentée et celle de la vesce diminuée. L'avoine est donc un compétiteur apte à exploiter plus que sa part des facteurs de l'environnement, tandis que la vesce est déprimée parce qu'elle n'est apte à recevoir et utiliser qu'une faible part seulement de lumière, d'eau et de nutriments.

Pour l'avoine (fig $1 \mathrm{a}, \mathrm{b}$ ), l'effet bénéfique engendré par la présence de la vesce, sur la biomasse par plante, est similaire à un effet densité. Pour préciser cet effet de l'association sur les performances des 2 espèces, on a représenté (fig 2) les courbes de croissance de l'avoine associée ( $a$ et $b$ ) et celles de la vesce en association ( $c$ et $d$ ) en même temps que les courbes des demi-densités correspondantes en culture pure (ex Va16 et Vp8); les valeurs pour les den-
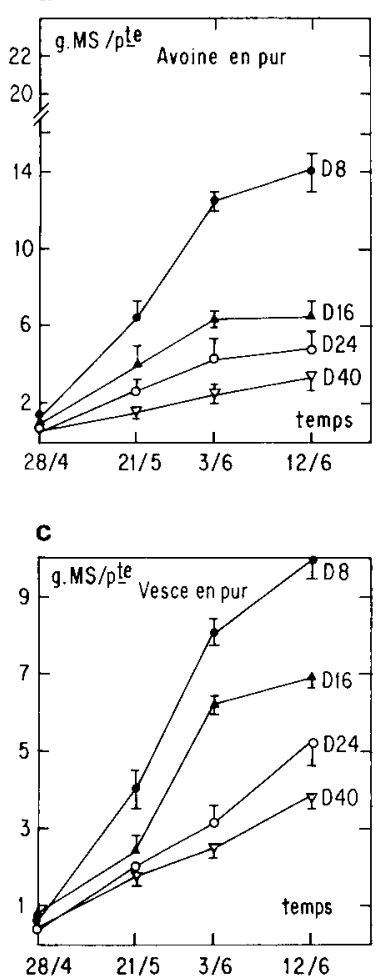

b

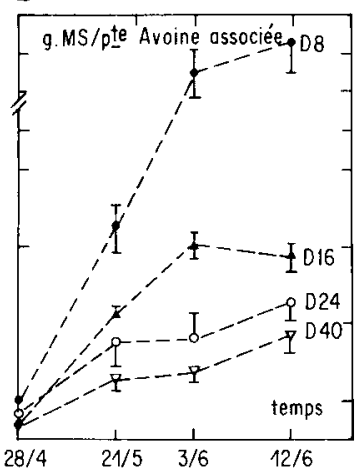

d

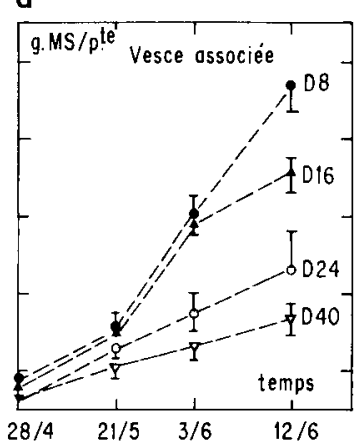

Fig 1. Évolution de la biomasse sèche par plante d'avoine (a et b) et de vesce (c et d). Variation avec la densité de peuplement et le mode de culture. - - : culture pure; - - - : association; D : densité totale en nombre de plantes/pot. 
sités 12 et 20 ont été calculées par interpolation à partir des densités 8 et 16 et 16 et 24 , respectivement...

Pour la vesce (fig 2c, d), il ressort, globalement, qu'à partir du stade montaison de l'avoine (21/5), sa biomasse en culture associée est nettement inférieure à celle de sa demi-densité en culture pure. Entre les stades montaison et gonflement de la graminée, la chute de production de biomasse engendrée par le compétiteur avoine est supérieure à celle engendrée par une augmentation de la densité en culture pure. Si l'on se reporte aux courbes de réponse de la biomasse (fig $1 \mathrm{c}$ ), on constate que, pour la vesce, à la date du 12/6 (état final), le rendement moyen des plantes dans l'association à la densité 8 (Va8) est de 8,42 g/plante. Ce poids est voisin de celui obtenu, en culture pure, avec 12 plantes par pot $(8,42 \mathrm{~g} /$ pte).

Ces effets densité et association sont bien mis en évidence quand on calcule les fonctions de réponse de la biomasse à la densité de chaque partenaire (tableau I). L'effet interspécifique est
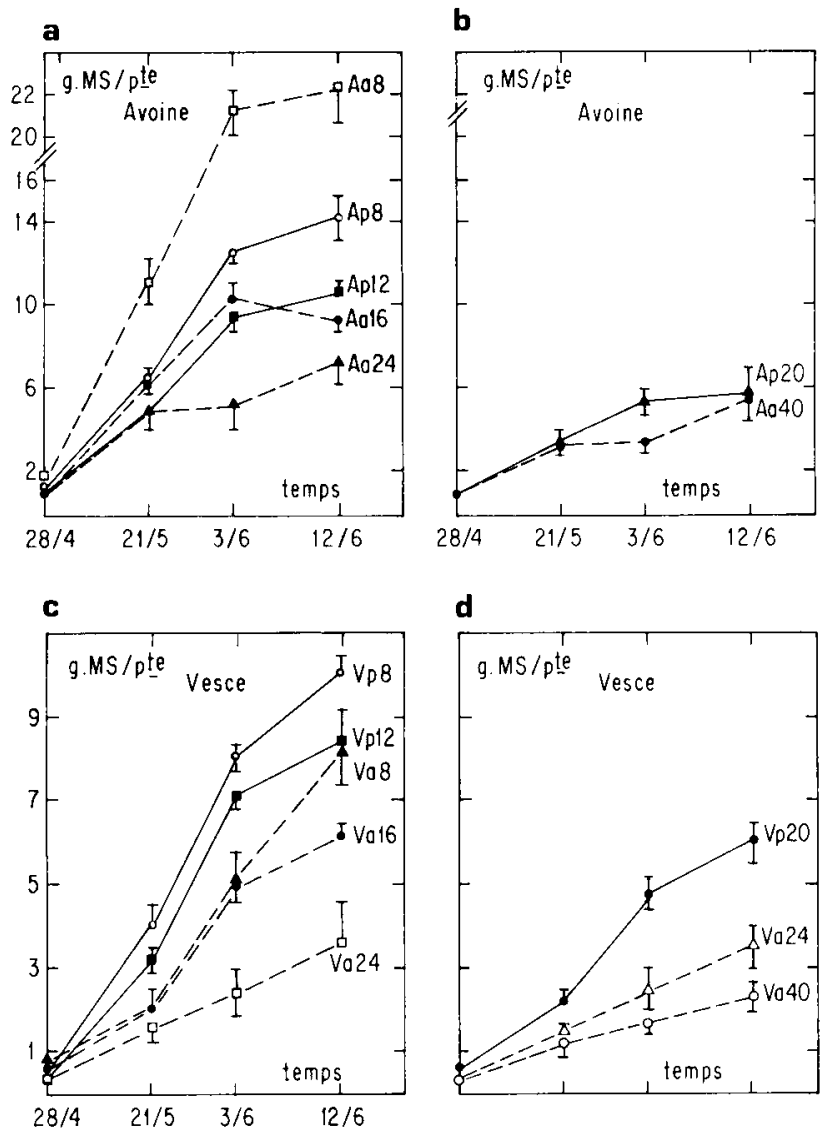

Fig 2. Effet de la présence du partenaire sur la biomasse par plante d'avoine ( $a$ et $b$ ) et de vesce ( $c$ et d). Comparaison des performances réalisées en association par rapport à la demi-densité correspondante en culture pure. $V \mathrm{pi}$ : vesce pure; Vai : vesce associée; Api : avoine pure; Avi : avoine associée; I : densité en nombre de plantes/pot. — : culture pure; - - : association. beaucoup plus déterminant que l'effet intraspécifique, pour la vesce. Dès le 28 avril, un individu d'avoine est perçu, par la vesce, comme 1,17 individu de vesce; l'intensité de la compétition interspécifique, due à l'avoine, atteint son maximum au 21 mai pour se stabiliser autour des 3 et 12 juin ( $a_{i j} / a_{i j}=1,75$ au 3 juin et 1,70 au 12 juin). Par contre, les 28 avril et 21 mai surtout, l'avoine ne répond qu'à sa propre densité, c'est-à-dire à la compétition intraspécifique; par la suite, un individu de vesce n'est perçu par l'avoine que comme 0,4 individu d'avoine.

\section{Effet du régime hydrique}

Les 2 espèces ont réagi de la même manière à la réduction de l'offre en eau du milieu (fig 3 ). La biomasse individuelle a été affectée de façon significative par le régime hydrique.

Pour une densité donnée, la matière sèche est d'autant plus faible que l'offre en eau est réduite. Quand l'intensité de la contrainte est élevée, la densité et le mode de culture (association) ont peu d'influence sur la biomasse des plants d'avoine, espèce pour laquelle le taux de substitution varie de 0,38 à 0,59 (tableau I). Par contre, la vesce réagit fortement à la compétition interspécifique, notamment lorsque l'offre en eau est
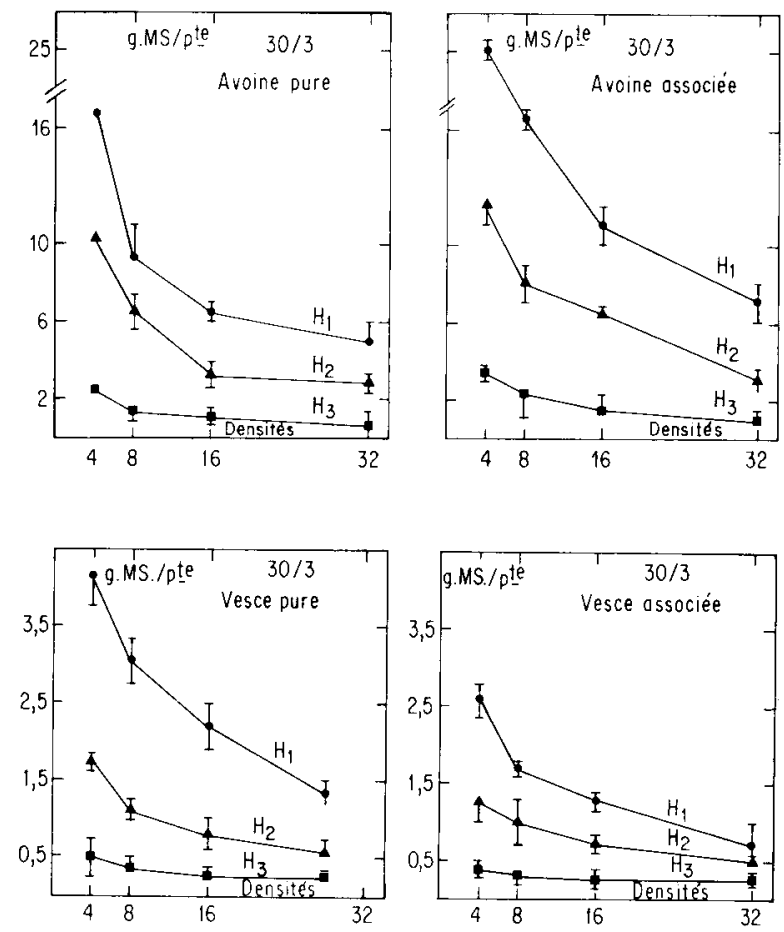

Fig 3. Évolution de la biomasse par plante d'avoine et de vesce avec la densité de peuplement. Variation en fonction du régime hydrique et du mode de culture; $\bullet$ : régime $\mathrm{H}_{1} ; \boldsymbol{\Delta}$ : régime $\mathrm{H}_{2} ; \mathbf{\square}$ : régime $\mathrm{H}_{3} ; \mathrm{D}$ : densité en nombre de plantes/ pot. 
Tableau I. Équation de réponse de la biomasse $(w)$ à la densité (d) de chaque partenaire (A : avoine, V : vesce).

\begin{tabular}{|c|c|c|c|c|}
\hline Date & Espèce & $W=f($ temps $)$ & $R^{2}$ & $a_{i j} / a_{i i}$ \\
\hline 28 avril & $\begin{array}{l}A \\
V\end{array}$ & $\begin{array}{l}w_{A}=1,63-0,0258 d_{A}-0,0025 d_{B} \\
w_{V}=0,88-0,0141 d_{V}-0,0165 d_{A}\end{array}$ & $\begin{array}{l}0,62 \\
0,70\end{array}$ & $\begin{array}{l}0,10 \\
1,17\end{array}$ \\
\hline 21 mai & $\begin{array}{l}A \\
\text { V }\end{array}$ & $\begin{array}{l}w_{A}=8,78-0,201 d_{A}-0,0240 d_{V} \\
w_{V}=3,06-0,0286 d_{V}-0,0838 d_{A}\end{array}$ & $\begin{array}{l}0,71 \\
0,58\end{array}$ & $\begin{array}{l}0,11 \\
2,93\end{array}$ \\
\hline 3 juin & $\begin{array}{l}A \\
\text { V }\end{array}$ & $\begin{array}{l}w_{A}=15,83-0,4239 d_{A}-0,1710 d_{V} \\
w_{V}=7,56-0,1266 d_{V}-0,2211 d_{A}\end{array}$ & $\begin{array}{l}0,64 \\
0,77\end{array}$ & $\begin{array}{l}0,40 \\
1,75\end{array}$ \\
\hline 12 juin & $\begin{array}{l}A \\
V\end{array}$ & $\begin{array}{l}w_{A}=16,83-0,4237 d_{A}-0,1782 d_{V} \\
w_{V}=9,72-0,1563 d_{V}-0,2653 d_{A}\end{array}$ & $\begin{array}{l}0,61 \\
0,89\end{array}$ & $\begin{array}{l}0,42 \\
1,70\end{array}$ \\
\hline Régime & Espèce & $w=f($ régime hydrique $)(\mathrm{Hi})$ & $R^{2}$ & $a_{i j} / a_{i i}$ \\
\hline $\mathrm{H}_{1}$ & $\begin{array}{l}A \\
V\end{array}$ & $\begin{array}{l}w_{A}=18,90-0,5366 d_{A}-0,2025 d_{V} \\
w_{V}=3,25-0,0561 d_{V}-0,1296 d_{A}\end{array}$ & $\begin{array}{l}0,64 \\
0,71\end{array}$ & $\begin{array}{l}0,38 \\
2,31\end{array}$ \\
\hline $\mathrm{H}_{2}$ & $\begin{array}{l}A \\
V\end{array}$ & $\begin{array}{l}w_{A}=10,35-0,2849 d_{A}-0,1673 d_{V} \\
w_{V}=1,41-0,0283 d_{V}-0,0357 d_{A}\end{array}$ & $\begin{array}{l}0,76 \\
0,77\end{array}$ & $\begin{array}{l}0,59 \\
1,26\end{array}$ \\
\hline $\mathrm{H}_{3}$ & $\begin{array}{l}A \\
V\end{array}$ & $\begin{array}{l}w_{A}=2,66-0,0731 d_{A}-0,0334 d_{V} \\
w_{V}=0,42-0,0059 d_{V}-0,0076 d_{A}\end{array}$ & $\begin{array}{l}0,69 \\
0,57\end{array}$ & $\begin{array}{l}0,46 \\
1,29\end{array}$ \\
\hline
\end{tabular}

optimale : $a_{i j} / a_{i j}=2,31$ pour $\mathrm{H}_{1}$ (ce qui signifie qu'un individu d'avoine est alors perçu comme 2,31 individus de vesce).

\section{Effet de la déficience minérale ( $\mathrm{K}$ et $\mathrm{NO}_{3}^{-}$)}

Dans le cas de contrainte nutritionnelle, la diminution du potassium dans la solution a un effet d'autant plus marqué sur la production de biomasse par plante (fig 4) que le niveau d'azote est élevé. L'association a toujours un effet bénéfique sur l'avoine et un effet déprimant sur la vesce, dans cette expérience où la densité est constante.

\section{Répartition de la biomasse}

\section{Vesce}

La répartition des assimilats dans les parties végétatives dépend du stade de développement de la plante et du niveau de stress, lequel favorise une accumulation de photosynthétats dans l'organe qui permet à la plante d'échapper à la contrainte.
Les 2 espèces répartissent leurs ressources selon des modalités différentes. L'avoine les investit surtout dans l'expansion de sa surface foliaire, alors que la vesce distribue sa biomasse dans un nombre moyen élevé de feuilles/plante et dans la longueur de l'axe principal.

En réponse à une déficience en potassium du milieu, la plante modifie la distribution de sa biomasse entre les différents organes (fig 5), selon le niveau d'azote et selon qu'elle est en culture
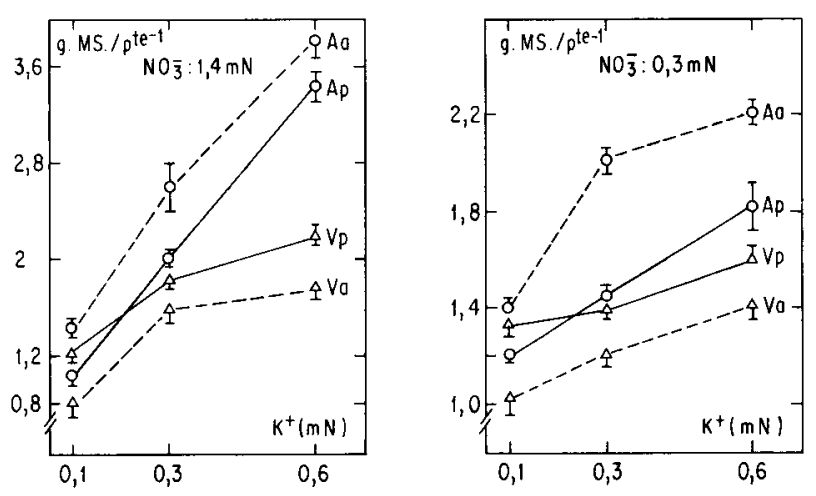

Fig 4. Variation de la biomasse sèche par plante d'avoine (A) et de vesce (V), en culture pure (Ap et $V p$ ) et en association ( $\mathrm{Aa}$ et $\mathrm{Va}$ ), avec l'appauvrissement du milieu du potas$\operatorname{sium}(0,6,0,3$ et $0,1 \mathrm{mN}$ de $\mathrm{K})$ et azote $(0,3$ et $1,4 \mathrm{mN}$ de $\mathrm{NO}_{3}^{-}$. 
pure ou en association avec l'avoine. À $0,3 \mathrm{mN}$ de $\mathrm{NO}_{3}^{-}$, le niveau de potassium en culture pure ne modifie pas la répartition de la matière sèche : feuilles $38 \%$, tiges $34 \%$ et racines $28 \%$. Quand le niveau d'azote augmente, l'effet du potassium est très hautement significatif $(P=$ $0,001)$. La quantité relative attribuée aux racines augmente avec la diminution du potassium dans la solution nutritive à $1,4 \mathrm{mN}$. Par rapport à la plante entière, la biomasse affectée aux racines est de 24,34 et $44 \%$ respectivement pour 0,6 , 0,3 et $0,1 \mathrm{mN}$ de $\mathrm{K}$. Cette augmentation du système racinaire se fait au détriment des feuilles dont la proportion régresse : 42,36 et $26 \%$ respectivement pour $0,6,0,3$ et $0,1 \mathrm{mN}$ de $\mathrm{K}$. En association avec l'avoine, la distribution de la biomasse est fortement modifiée. L'effet partenaire se traduit par une augmentation des tiges et des racines et une diminution des feuilles.

A 1,4 mN d'azote, les quantités attribuées aux feuilles ne sont que de 20,12 et $8 \%$ et, pour les racines 34,44 et $50 \%$ respectivement pour 0,6 ,
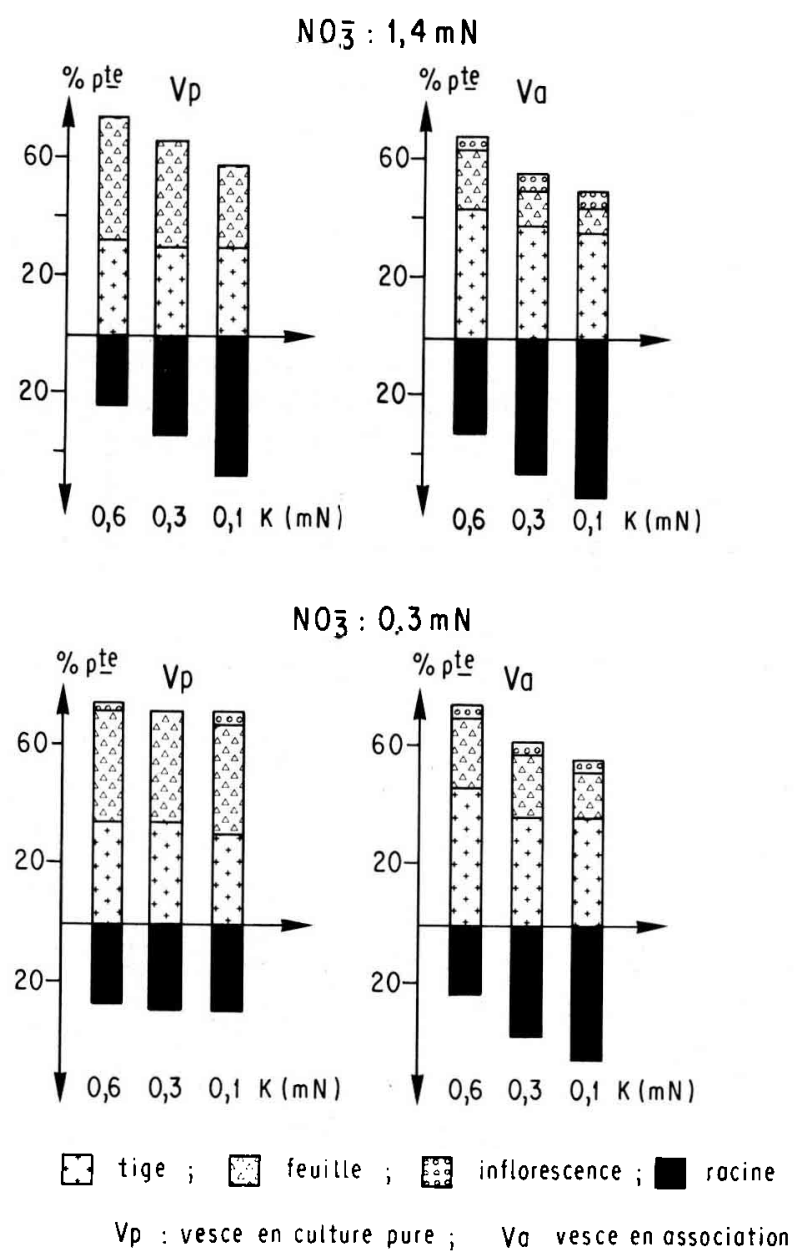

Fig 5. Effet du potassium sur la répartition de la biomasse sèche par plante de vesce en culture pure et en association à 2 niveaux d'azote $\left(0,3\right.$ et $1,4 \mathrm{mN}$ de $\left.\mathrm{NO}_{3}^{-}\right)$
0,3 et $0,1 \mathrm{mN}$ de $\mathrm{K}$. Sur le plan agronomique, cette modification engendrée par la présence de l'avoine va se traduire par une diminution de la valeur nutritive. La richesse en éléments nutritifs est liée au rapport feuille sur tige.

\section{Avoine}

Pour l'avoine en culture pure, le même phénomène que celui décrit précédemment se produit, avec toutefois une plus grande partie allouée aux feuilles (fig 6). À un niveau d'azote supérieur $(1,4 \mathrm{mN})$, la quantité attribuée aux racines en culture pure augmente avec la diminution du potassium. À $0,1 \mathrm{mN}$ de potassium, la proportion des racines dépasse $50 \%$. Quand on rapporte la variation des masses racinaires à celle des parties aériennes sèches $(\mathrm{Ra} / \mathrm{Pa})$, on illustre cette augmentation des racines sous l'influence d'une déficience potassique. En effet, le rapport biomasse des racines sur biomasse des parties aériennes pour l'avoine associée à $0,3 \mathrm{mN}$ d'azote
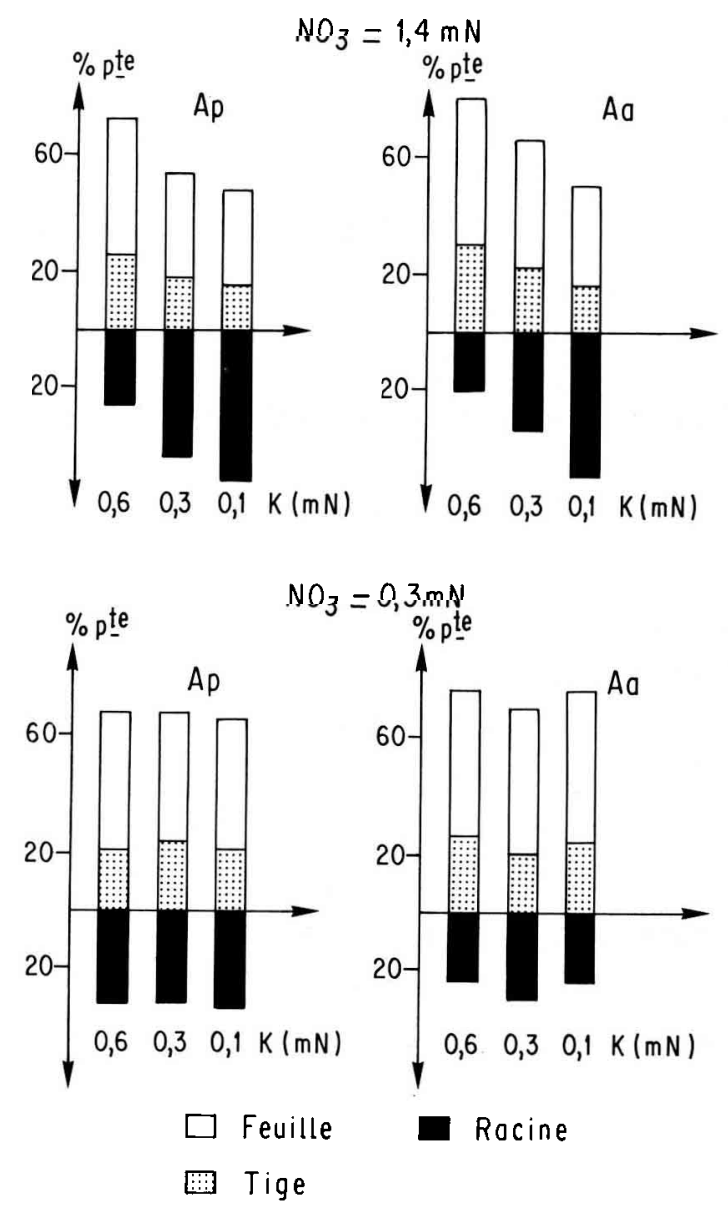

Fig 6. Effet du potassium et de l'azote sur la répartition de la biomasse sèche par plante d'avoine en culture pure et en association. 
est de $0,23,0,47$ et 0,67 respectivement pour $0,6,0,3$ et $0,1 \mathrm{mN}$ de $\mathrm{K}$. La valeur du rapport diminue avec le niveau d'azote : à $0,3 \mathrm{mN}$ d'azote, le rapport racines sur parties aériennes est de $0,09,0,15$ et 0,22 respectivement pour $0,6,0,3$ et $0,1 \mathrm{mN}$ de $\mathrm{K}$.

En association, chez l'avoine, la présence de la vesce s'accompagne d'une diminution de la part de biomasse attribuée aux racines et d'une augmentation de la biomasse foliaire. En culture pure, la répartition se fait au profit des racines pour les traitements à niveau d'azote élevé et déficients en potassium.

\section{DISCUSSION ET CONCLUSION}

Les effets d'interférence produits sur la croissance des vesces par la présence des avoines pendant la durée de la culture montrent qu'à densité numérique par espèce équivalente, la production par plante d'avoine augmente et celle de la vesce diminue par rapport à la culture pure. L'effet bénéfique observé sur l'avoine est d'autant plus marqué que le niveau de contrainte hydrique est moindre (et inversement pour $N$ ). Cet effet, sur avoine, est similaire, en début de cycle, à un effet densité (même comportement que sa demi-densité en culture pure). La densité de peuplement est un facteur de compétition qui détermine la production par unité de surface.

Contrairement à l'avoine, la biomasse de la vesce résulte surtout d'une compétition interspécifique. La densité a affecté l'ensemble des paramètres de la morphogénèse et de la démographie des organes (Ouknider et al, 1991). Sous l'effet de l'augmentation de l'intensité du déficit hydrique, la densité semble avoir moins d'influence.

En association, les 2 espèces répartissent leurs ressources selon des modalités différentes. L'avoine les investit surtout dans l'expansion de sa surface foliaire, alors que la vesce distribue sa biomasse dans un nombre moyen élevé de feuilles par plante et dans la longueur de l'axe principal.

En conditions de déficience en potassium, la biomasse individuelle est répartie différemment entre les organes selon l'espèce et le mode de culture. En culture pure de vesce, le niveau de potassium à $0,3 \mathrm{mN}$ de $\mathrm{NO}_{3}^{-}$ne modifie pas la répartition de la biomasse. Par contre, à niveau d'azote élevé $(1,4 \mathrm{mN})$, la biomasse racinaire augmente, au détriment des feuilles, avec la diminution de l'offre en potassium.
Soumise à la gêne occasionnée par son voisin (avoine), la vesce réagit par une augmentation de la proportion répartie dans les tiges, et, dans une moindre mesure, aux racines et une diminution de la proportion de feuilles. Sur trèfle violet, Jacquard et al (1985) ont montré qu'en association l'investissement des ressources s'effectue au profit des pétioles ( $47 \%$ ) et que dans le cas de la concurrence intraspécifique les tiges représentent $32 \%$. D'autres auteurs ont rapporté que la proportion de la biomasse localisée dans les racines augmente avec l'aridité du milieu (Struik et Bray, 1970; Hickman, 1977). Le même effet s'observe en cas de déficience minérale (Chapin, 1980).

Le rapport racines sur parties aériennes dépend surtout des espèces et des conditions initiales de croissance. Le stade de développement et le niveau du stress favorisent l'accumulation de biomasse dans l'organe qui permet à la plante soit d'échapper à la contrainte soit d'assurer sa survie. Katherine et al (1979) ont étudié le modèle de répartition de biomasse chez 8 espèces et ils ont conclu qu'une répartition aux racines d'environ $20 \%$ de la biomasse totale représente le minimum nécessaire à une croissance optimale.

\section{RÉFÉRENCES}

Chapin FS (1980) The mineral nutrition of wild plants. Annu Rev Ecol Syst 11, 233-260

Connolly $J(1987)$ On the use of response models in mixture experiments. Oecologia 72, 95-103

Fowler N (1982) Competition and coexistence in a North Carolina grassland. III. Mixtures of component species. $J$ Ecol 70, 77-92

Grime JP (1977) Evidence for the existence of three primary strategies in plants and its relevance to ecological and evolutionary theory. Am Nat 111, 1169-1194

Hickman JC (1977) Energy allocation and niche differentiation in four co-existing annuals species of $\mathrm{PO}$ lygonum in Western North America. J Ecol 65, 317326

Hickman JC, Pitelka LF (1975) Dry weight indicates energy allocation in ecological strategy analysis of plants. Oecologia 21, 112-121

Jacquard $P$, Ibn Tatou M, Maitre JP, Al Sbei R (1985) Allocation des ressources chez quelques végétaux : facteurs de variation. Bull Ecol 16, 35-46

Katherine LB, Hermi DH, Niles WE (1979) Seasonal changes in biomass allocation in eight winter annuals of the Mojave desert. J Ecol 67, 781-787 
Ouknider M, Jacquard P, Elkhyari D (1991) Concurrence chez Vicia sativa $L$ et Avena sativa L. I. Effets des contraintes hydriques et nutritionnelles sur la morphogenèse et la démographie des organes. Agronomie 11, 447-456

Peterson DL, Bazzaz FA (1978) Life cycle characteristics of Aster pilosa in early successional habitats. Ecology 59, 1005-1013

Pickett STA, Bazzaz FA (1978) Organisation of an assemblage of early successional species on a soil moisture gradient. Ecology 59, 1248-1255

Struick GJ, Bray JR (1970) Root-shoot ratios of native forest herbs and Zea mays at different soil-moisture levels. Ecology 51, 892-893

Thomas H (1984) Effects of drought on growth and competitive ability of perennial ryegrass and white clover. J App/ Ecol 21, 591-602

Turkington R, Harper JL (1979) The growth, distribution and neighbour relationships of Trifolium repens in a permanent pasture. IV. Fine-scale biotic differentiation. J Eco/67, 245-254

Troughton A (1977) Relationship between the root and the shoot systems of grasses. In: The below-ground ecosystem: a synthesis of plant-associated processes (JK Marshall, ed). Range Science Department, Science Series $n^{\circ} 26$, Colorado State University, Fort Collins, 39-51

Watkinson AR (1982) Factors affecting the density response of Vulpia fasciculata. J Ecol 70, 149-161

Werner PA, Platt WJ (1976) Ecological relationships of co-occurring goldenrods (Solidago: compositae). Am Nat 110, 959-971

Winner WE, Mooney HA, Williams $\mathrm{K}$, Von Caemmerer $\mathrm{S}$ (1985) Measuring and assessing $\mathrm{SO}_{2}$ effects on photosynthesis and plant growth. In: Sulfur dioxide and vegetation. Physiology, ecology and policy issues (WE Winner, JA Mooney, RA Goldstein, eds). Stanford Univ Press, Stanford, CA, 118-132 